\title{
Optimal Dextrose Concentration for Pain Control in Healthy Newborns during Hepatitis B Vaccination
}

\author{
Seong Hee Oh, In Ah Kim, and Hyunseung Jin \\ Department of Pediatrics, Gangneung Asan Hospital, University of Ulsan College of Medicine, Gangneung, Korea
}

\section{ABSTRACT}

Purpose: Glucose has been recommended as an analgesic for mild to moderately painful procedures in neonates. The goal of this study was to assess the optimal dextrose concentration for pain control in newborns.

Methods: This prospective, randomized, blinded clinical trial included 116 healthy fullterm newborns. The neonates were randomly assigned to the following four groups by drawing straws: groups receiving sterile water or a $10 \%, 20 \%$, or $40 \%$ dextrose solution orally. Each group was treated with the assigned solution prior to hepatitis B vaccination. The Neonatal Facial Coding System (NFCS) and the Neonatal Infant Pain Scale (NIPS) scores were evaluated before, immediately after, and 2 minutes after the injection in all neonates. Premature Infant Pain Profile (PIPP) scores were evaluated during the injection. All procedures were video-recorded, and pain scores were assessed by two independent observers who were not involved in the care of the newborns studied. The pain scores were compared among the four groups.

Results: The $40 \%$ dextrose solution significantly reduced the NFCS $(P=0.002)$ and the PIPP scores $(P=0.001)$ compared with sterile water. No hyperglycemic events were noted in the study subjects 2 hours after the injection.

Conclusion: The $40 \%$ dextrose solution effectively relieved pain due to intramuscular injection in full-term newborns without causing hyperglycemic events. However, the $10 \%$ and $20 \%$ dextrose solutions did not affect neonatal pain scores.

Key Words: Glucose, Pain management, Newborn

\section{INTRODUCTION}

Repeated exposure to pain during infancy has been reported to be related to adverse neurodevelopmental outcomes that have both immediate and long-term effects ${ }^{1-3)}$. Thus, the American Academy of Pediatrics and the Canadian Pediatric Society suggested the routine assessment of pain, minimizing the number of painful procedures, and managing pain using both pharmacologic and non-pharmacologic methods ${ }^{4,5)}$. Witt et al. ${ }^{6)}$ also recommended the use of tiered anesthesia during painful procedures. The use of sweet solutions such as sucrose or glucose were recommended for mild to moderately painful procedures,
Received: 26 January 2018

Revised: 10 May 2018

Accepted: 15 May 2018

Correspondence to: Hyunseung Jin Department of Pediatrics, Gangneung Asan Hospital, University of Ulsan College of Medicine, 38 Bangdong-gil, Gangneung 25440, Korea

Tel: +82-33-610-3168

Fax: +82-33-641-8148

E-mail: childlove72@naver.com

Copyright(c)

By Korean Society of Neonatology.

All right reserved.

This is an Open-Access article distributed under the terms of the Creative Commons Attribution Non-Commercial License (http://creativecommons.org/licenses/ by-nc/4.0), which permits unrestricted non-commercial use, distribution, and reproduction in any medium, provided the original work is properly cited. 
as a sweet solution with non-nutritive sucking, skin-to-skin contact, and breastfeeding have reported synergistic effects ${ }^{7-9)}$.

The use of a $24 \%$ sucrose solution is usually recommended for neonatal pain control, although the exact oral dose has not been determined $^{10)}$. A recent meta-analysis of 38 studies reported that a $20 \%$ to $30 \%$ glucose solution was also effective against pain ${ }^{11)}$. However, only a few studies included in this meta-analysis compared different glucose concentrations, and pain scores were not systematically analyzed.

This study aimed to assess the optimal concentration of dextrose for providing pain control in newborns. We evaluated the Neonatal Facial Coding System (NFCS) ${ }^{12)}$, Neonatal Infant Pain Scale (NIPS) $)^{13)}$, and Premature Infant Pain Profile (PIPP) ${ }^{14)}$ scores after the oral administration of sterile water or a $10 \%, 20 \%$, or $40 \%$ dextrose solution prior to hepatitis B vaccination.

\section{MATERIALS AND METHODS}

This prospective, randomized, controlled, blinded clinical trial was conducted in the nursery of Gangneung Asan Hospital of Korea between January and October 2013. Written informed consent was provided by the parents of each subject prior to enrollment and the study was approved by the hospital's Institutional Review Board (GNAH IRB No. 2013-044).

\section{Subjects and randomization}

Full-term newborn infants born at $\geq 37$ weeks' gestation with a birth weight $\geq 2,500$ g were eligible to participate. Newborns with 1- or 5-minute Apgar scores $<7$ or those who needed close monitoring including pulse oximetry or repeated serum glucose levels were excluded. The newborns were randomly allocated, by drawing straws, to receive sterile water or $10 \%, 20 \%$, or $40 \%$ dextrose prior to the administration of the vaccine.

\section{Intervention}

Each participant was orally administered $1 \mathrm{~mL}$ of sterile water or $10 \%, 20 \%$, or $40 \%$ dextrose solution. Each solution was administered dropwise on the anterior part of the tongue with a 5-mL syringe to prevent any effect from sucking action. Two minutes later, the hepatitis B vaccine was administered to the thigh as an intramuscular injection of $0.5 \mathrm{~mL}$ using a 25-gauge needle (Euvax ${ }^{\circledR}$, LG Life Sciences Ltd., Seoul, Korea). All procedures were performed when the newborns remained stable after more than 3 hours after birth to rule out the effect of birth-related conditions. We used no other non-pharmacologic analgesic methods such as non-nutritive sucking or skin-to-skin contact.

\section{Pain monitoring and assessment}

All interventions and physical appearance of newborns including facial expression and movement were video-recorded from the time of administration of the oral solution to 2 minutes after vaccination. Heart rates and oxygen saturation were monitored by a pulse oximeter placed on the left foot and video-recorded simultaneously. Two researchers who did not participate in the procedures, assessed pain scores separately, by analyzing the video recordings. The degree of pain was evaluated using the NFCS, NIPS, and PIPP scales in all groups. The NFCS and NIPS scores were assessed three times: before the injection (baseline), immediately after the injection (injection), and 2 minutes after the injection (recovery). We analyzed change in scores from baseline to injection and from baseline to recovery. The PIPP score was checked once during the injection. We calculated the mean of the scores assessed by the two researchers. The serum glucose level was checked at 2 hours after administration of the vaccine to assess hyperglycemia (defined as a serum glucose level >200 mg/dL).

\section{Statistical analysis}

Statistical analysis was performed using SAS version 9.4 (SAS Institute Inc., Cary, NC, USA). The changes in the NFCS and NIPS scores with different dextrose solutions were evaluated from baseline to injection and from baseline to recovery by repeated measures multivariate analysis of variance. The interaction between dextrose concentration and the onset time and the main effect of the dextrose concentration were evaluated. PIPP scores were evaluated by analysis of variance, while post hoc analysis was conducted by Bonferroni correction. $P$-values $<0.05$ were considered significant, while those $<0.016$ were subjected to Bonferroni correction.

\section{RESULTS}

A total of 120 newborns were enrolled and randomly assigned to four groups of 30 each. However, errors with video-recording occurred at three subjects in the sterile water group and one in the $10 \%$ dextrose group. Therefore, pain scores in 116 newborns 
Table 1. Characteristics of the Newborns

\begin{tabular}{|c|c|c|c|c|}
\hline Characteristic & $\begin{array}{l}\text { Sterile water } \\
\quad(\mathbf{n}=27)\end{array}$ & $\begin{array}{c}10 \% \text { Dextrose } \\
(n=29)\end{array}$ & $\begin{array}{c}20 \% \text { Dextrose } \\
(n=30)\end{array}$ & $\begin{array}{c}40 \% \text { Dextrose } \\
(n=30)\end{array}$ \\
\hline Gestational age (wks) & $39.2 \pm 6.2$ & $38.8 \pm 7.0$ & $39.0 \pm 7.1$ & $38.7 \pm 7.0$ \\
\hline Birth weight (g) & $3,244.6 \pm 250.8$ & $3,248.2 \pm 405.6$ & $3,182.2 \pm 327.9$ & $3,181.1 \pm 372.1$ \\
\hline \multicolumn{5}{|l|}{ Apgar score } \\
\hline $1 \mathrm{~min}$ & $8.5 \pm 0.8$ & $8.3 \pm 0.9$ & $8.5 \pm 0.6$ & $8.3 \pm 0.8$ \\
\hline $5 \mathrm{~min}$ & $9.6 \pm 0.6$ & $9.5 \pm 0.7$ & $9.6 \pm 0.5$ & $9.6 \pm 0.6$ \\
\hline Cesarean section* & $12(44.4)$ & $24(83.8)$ & $17(56.7)$ & $21(70.0)$ \\
\hline Time to vaccination after birth (min) & $220.2 \pm 48.9$ & $239.5 \pm 48.4$ & $222.8 \pm 41.7$ & $238.1 \pm 80.8$ \\
\hline Time to vaccination after feeding ( $\mathrm{min}$ ) & $91.5 \pm 57.6$ & $76.8 \pm 52.8$ & $94.8 \pm 49.3$ & $73.5 \pm 58.4$ \\
\hline Serum glucose at 2 hours after vaccination $(\mathrm{mg} / \mathrm{dL})$ & $63.4 \pm 10.6$ & $63.4 \pm 8.6$ & $68.2 \pm 9.8$ & $67.4 \pm 13.3$ \\
\hline
\end{tabular}

Values are expressed as mean \pm standard deviation or number (\%).

${ }^{*} P$-value $=0.01$ for difference in cesarean section among the four groups.

were included in the final analysis: 27 in the sterile water group, 29 in the $10 \%$ dextrose group, 30 in the $20 \%$ dextrose group, and 30 in the $40 \%$ dextrose group.

The mean gestational age was $38.9 \pm 6.9$ weeks and the mean birth weight, $3,212.9 \pm 342.6 \mathrm{~g}$. The procedures were performed at a mean of $230.3 \pm 57.2$ minutes after birth and $84.3 \pm 54.5$ minutes after the most recent feed. There was no significant difference in clinical characteristics between the groups except the mode of delivery (Table 1). There was no difference in the mean serum glucose level checked 2 hours after injection among groups, and no hyperglycemic events were observed.

Tables 2, 3 present the mean NFCS, NIPS, and PIPP scores assessed by the two observers at each time point during the procedure, with different dextrose concentrations. Interobserver reliability showed good agreement; the intraclass correlations (ICC) were $\geq 0.8$ for NFCS scores at recovery, NIPS scores at baseline and recovery, and PIPP scores compared to $\geq 0.6$ for the other parameters.

We compared the changes in NFCS and NIPS scores from baseline to injection and those from baseline to recovery between groups. The trend in change of scores were similar between groups in both NFCS $(P=0.284)$ and NIPS $(P=0.166)$. The $40 \%$ dextrose group showed significantly lower NFCS scores compared to the sterile water group regardless of the time of evaluation $(P=$ 0.002), while there was no difference in the change of NIPS scores between groups. The $10 \%$ and $20 \%$ dextrose groups showed no differences compared to the sterile water group on either pain scale (Table 4). Furthermore, PIPP scores showed a significant difference only between the sterile water and the $40 \%$ dextrose groups $(P=0.001)$ (Table 3$)$.
Table 2. NFCS and NIPS Scores at Baseline, Injection, and Recovery

\begin{tabular}{lcccc}
\hline & $\begin{array}{c}\text { Sterile } \\
\text { water } \\
(\mathrm{n}=27)\end{array}$ & $\begin{array}{c}10 \% \\
\text { Dextrose } \\
(\mathrm{n}=29)\end{array}$ & $\begin{array}{c}20 \% \\
\text { Dextrose } \\
(\mathrm{n}=30)\end{array}$ & $\begin{array}{c}40 \% \\
\text { Dextrose } \\
(\mathrm{n}=30)\end{array}$ \\
\hline NFCS & & & & \\
Baseline & $0.6 \pm 1.4$ & $0.5 \pm 0.6$ & $0.5 \pm 0.4$ & $0.5 \pm 0.8$ \\
Injection & $6.6 \pm 1.5$ & $6.4 \pm 1.2$ & $6.3 \pm 1.1$ & $5.8 \pm 1.0$ \\
Recovery & $2.0 \pm 2.7$ & $1.8 \pm 2.6$ & $0.9 \pm 1.5$ & $0.5 \pm 1.1$ \\
NIPS & & & & \\
Baseline & $0.6 \pm 1.3$ & $0.9 \pm 1.0$ & $0.5 \pm 0.5$ & $0.5 \pm 0.6$ \\
Injection & $5.5 \pm 1.3$ & $5.3 \pm 1.1$ & $5.3 \pm 1.0$ & $4.8 \pm 1.1$ \\
Recovery & $1.8 \pm 2.3$ & $1.7 \pm 2.3$ & $0.9 \pm 1.2$ & $0.5 \pm 1.0$ \\
\hline
\end{tabular}

Values are expressed as mean \pm standard deviation.

Abbreviations: NFCS, Neonatal Facial Coding System; NIPS, Neonatal Infant Pain Scale.

Table 3. Comparison of PIPP scores

\begin{tabular}{lccc}
\hline & PIPP scores & $P$-value* & $P_{\text {-value }}^{\dagger}$ \\
\hline Sterile water $(\mathrm{n}=27)$ & $12.3 \pm 2.6$ & 0.001 & Reference \\
10\% Dextrose $(\mathrm{n}=29)$ & $10.3 \pm 2.9$ & & 0.062 \\
$20 \%$ Dextrose $(\mathrm{n}=30)$ & $11.2 \pm 2.8$ & & 0.951 \\
$40 \%$ Dextrose $(\mathrm{n}=30)$ & $9.3 \pm 2.7$ & & 0.001 \\
\hline
\end{tabular}

Values are expressed as mean \pm standard deviation.

*Analysis of variance (ANOVA); ${ }^{\dagger}$ Post hoc analysis by Bonferroni correction considered statistically significant at $P<0.016$.

Abbreviation: PIPP, Premature Infant Pain Profile.

\section{DISCUSSION}

This study showed that the administration of $40 \%$ dextrose prior to hepatitis B vaccination effectively relieved pain compared to sterile water on the NFCS and PIPP scales without any 
Table 4. Comparison of Change in Score from Baseline to Injection and from Baseline to Recovery According to the NFCS and NIPS

\begin{tabular}{|c|c|c|c|c|c|c|c|}
\hline & \multicolumn{4}{|c|}{ NFCS scores } & \multicolumn{3}{|c|}{ NIPS scores } \\
\hline & $\Delta$ Injection* & $\Delta$ Recovery $^{\dagger}$ & $P$-value ${ }^{\ddagger}$ & $P$-value ${ }^{\S}$ & $\Delta$ Injection* & $\Delta$ Recovery $^{\dagger}$ & $P$-value ${ }^{\ddagger}$ \\
\hline Sterile water $(\mathrm{n}=27)$ & $6.1 \pm 2.0$ & $1.5 \pm 2.3$ & 0.007 & Reference & $4.9 \pm 2.0$ & $1.2 \pm 2.0$ & 0.062 \\
\hline 10\% Dextrose (n=29) & $5.8 \pm 1.3$ & $1.3 \pm 2.5$ & & 0.565 & $4.4 \pm 1.3$ & $0.9 \pm 2.2$ & \\
\hline $20 \%$ Dextrose $(\mathrm{n}=30)$ & $5.8 \pm 1.1$ & $0.4 \pm 1.4$ & & 0.068 & $4.8 \pm 1.1$ & $0.4 \pm 1.2$ & \\
\hline $40 \%$ Dextrose $(n=30)$ & $5.3 \pm 1.2$ & $0.0 \pm 1.4$ & & 0.002 & $4.3 \pm 1.2$ & $0.0 \pm 1.1$ & \\
\hline
\end{tabular}

Values are expressed as mean \pm standard deviation.

${ }^{*}$ Score changes from baseline to injection; ${ }^{\dagger}$ Score changes from baseline to recovery; ${ }^{*}$ Main effect of different concentration of dextrose analyzed by repeated measures multivariate analysis of variance; ${ }^{\circledR}$ Post hoc analysis by Bonferroni correction considered statistically significant at $P<0.016$.

Abbreviations: NFCS, Neonatal Facial Coding System; NIPS, Neonatal Infant Pain Scale.

hyperglycemic events. However, the $10 \%$ and $20 \%$ dextrose solutions had no significant analgesic effect on the neonatal pain scores.

Although the precise mechanism has not yet been determined, sweet taste is thought to have an analgesic effect by stimulating oro-gustatory receptors and release of endogenous opioids ${ }^{15,16)}$. A previous study showed that sweet taste is important to pain relief regardless of the carbohydrate content ${ }^{17)}$. Sucrose water is commonly used to reduce minor procedural pain in newborns; glucose has an equivalent analgesic effect ${ }^{11,18,19)}$. Glucose solution is easily available in the neonatal intensive care unit, whereas a sucrose solution must be prepared by the pharmacy or the nutrition team in advance because it is not commercially available in Korea. Accordingly, glucose can be used as an alternative to sucrose for providing analgesia in newborns.

Several previous randomized clinical trials have shown that a $\geq 20 \%$ glucose solution provides effective pain relief in newborns ${ }^{20-25)}$. A recent meta-analysis suggested that $20 \%$ to $30 \%$ glucose has an analgesic effect ${ }^{11)}$. However, in our study, a dextrose concentration of $20 \%$ or less did not demonstrate an analgesic effect; only the $40 \%$ dextrose solution revealed a statistically significant analgesic effect compared to sterile water.

Although $10 \%$ and $20 \%$ dextrose solutions were ineffective in reducing pain compared to sterile water, there was a positive relationship between the dextrose concentration and analgesic effect in our study. Thus, we believe that a concentration of $21 \%$ to $40 \%$ dextrose might be the cut-off range for the control of neonatal pain. In contrast, an earlier study reported that the analgesic effect plateaued off at a concentration of $25 \%$, based on the finding that both $25 \%$ and $50 \%$ glucose showed similar effect $^{20)}$. Blass and Shah ${ }^{16)}$ and Blass and Shide ${ }^{26)}$ also suggested that a sweet solution had a flat dose-response relationship. We did not study a dextrose concentration between $20 \%$ to $40 \%$. Dex- trose concentrations between $20 \%$ to $40 \%$. need to be included in further studies to assess the dose-response relationship and determine the exact cut-off range. The onset and duration of pain control with dextrose were not determined. Blass and Shah ${ }^{16)}$ concluded that the level of endogenous opioids peaked 2 minutes after feeling the sensation of sweetness and lasted for 5 to 10 minutes. An earlier study showed that this effect peaked at 2 minutes after injection when there was a 2-minute interval between glucose administration and a painful procedure ${ }^{27)}$. Based on these studies, glucose was given orally 2 minutes before injection and pain scoring was done 2 minutes after the injection in our study. However, we believe that further studies are needed to investigate the ideal timing of glucose administration.

We used three pain scales — NFCS, NIPS, and PIPP — for this study. They have been used as reliable tools for assessment of procedural pain in previous studies ${ }^{5,6,11)}$. NFCS includes multiple indicators of facial expression in neonates and NIPS assesses facial expression, cry, breathing patterns, movements of arms and legs, and the state of arousal. PIPP is available for both preand full-term infants adjusted for gestational age. Objective indicators such as heart rate and oxygen saturation were used to assess the physiologic responses ${ }^{14)}$. Score of $\geq 3 / 8$ in the $\mathrm{NFCS}^{12)}$, $\geq 4 / 7$ in the NIPS ${ }^{13)}$, and $\geq 7 / 18-21$ (according to the gestational age) in the PIPP scales ${ }^{14)}$ are considered to indicate pain. Based on our results, the odds ratio for pain decreased significantly with increasing concentration of dextrose, as assessed by all three pain scales based on these cut-off values. However, we finally chose the repeated measures multivariate analysis of variance as we felt that this method was more relevant for this study.

This study has several limitations. First, as it was not possible to analyze the effect of various concentrations of dextrose, we could not find a more precise, narrower range than the $20 \%$ to $30 \%$ concentration of dextrose previously reported to be effective 
for analgesia. We found that $20 \%$ dextrose, which was effective in a previous study, was not an effective analgesic in this study. Second, the pain scoring system we used may be of limited utility. It is essential to assess pain accurately to analyze analgesic effect. The pain scales used in this study included subjective parameters and had fundamental limitations despite being commonly used. Although the ICC between the two researchers was good, they had difficulty scoring participants in exactly the same way.

However, this study also has several strengths. First, it was a prospective, randomized, controlled, blinded clinical trial. Second, the intensity of pain was assessed by three different pain scales; we could confirm similarities in assessment with the use of these scales. Third, to our knowledge, this is the first study to evaluate the analgesic effects of various concentrations of glucose using pain scales. Fourth, we video-recorded the procedure to ensure blinding of pain scoring.

In conclusion, $40 \%$ dextrose effectively relieved minor procedural pain in newborns without causing hyperglycemic events. There was no significant analgesic effect of $10 \%$ or $20 \%$ dextrose on the neonatal pain scores. Further studies are needed to determine the lowest concentration of dextrose between $21 \%$ to $40 \%$ that is most effective and has fewer complications. There is no well-structured protocol for neonatal pain control in Korea so far, and more studies are needed to develop guidelines based on accurate understanding and evidence.

\section{CONFLICT OF INTEREST}

No potential conflict of interest relevant to this article was reported.

\section{REFERENCES}

1) Vinall J, Grunau RE. Impact of repeated procedural pain-related stress in infants born very preterm. Pediatr Res 2014;75:584-7.

2) Doesburg SM, Chau CM, Cheung TP, Moiseev A, Ribary U, Herdman AT, et al. Neonatal pain-related stress, functional cortical activity and visual-perceptual abilities in school-age children born at extremely low gestational age. Pain 2013;154 1946-52.

3) Hermann C, Hohmeister J, Demirakca S, Zohsel K, Flor H. Long-term alteration of pain sensitivity in school-aged children with early pain experiences. Pain 2006;125:278-85.

4) American Academy of Pediatrics Committee on Fetus and Newborn; American Academy of Pediatrics Section on Surgery; Canadian Paediatric Society Fetus and Newborn Committee, Batton DG, Barrington KJ, Wallman C. Prevention and management of pain in the neonate: an update. Pediatrics 2006;118: 2231-41.

5) Committee on Fetus and Newborn and Section on Anesthesiology and Pain Medicine. Prevention and management of procedural pain in the neonate: an update. Pediatrics 2016;137: e20154271.

6) Witt N, Coynor S, Edwards C, Bradshaw H. A guide to pain assessment and management in the neonate. Curr Emerg Hosp Med Rep 2016;4:1-10.

7) Gibbins S, Stevens B, Hodnett E, Pinelli J, Ohlsson A, Darlington G. Efficacy and safety of sucrose for procedural pain relief in preterm and term neonates. Nurs Res 2002;51:375-82.

8) Chermont AG, Falcao LF, de Souza Silva EH, de Cassia Xavier Balda R, Guinsburg R. Skin-to-skin contact and/or oral $25 \%$ dextrose for procedural pain relief for term newborn infants. Pediatrics 2009;124:e1101-7.

9) Gradin M, Finnstrom O, Schollin J. Feeding and oral glucose: additive effects on pain reduction in newborns. Early Hum Dev 2004;77:57-65.

10) Stevens B, Yamada J, Ohlsson A, Haliburton S, Shorkey A. Sucrose for analgesia in newborn infants undergoing painful procedures. Cochrane Database Syst Rev 2016;7:CD001069.

11) Bueno M, Yamada J, Harrison D, Khan S, Ohlsson A, AdamsWebber T, et al. A systematic review and meta-analyses of nonsucrose sweet solutions for pain relief in neonates. Pain Res Manag 2013;18:153-61.

12) Grunau RV, Craig KD. Pain expression in neonates: facial action and cry. Pain 1987;28:395-410.

13) Lawrence J, Alcock D, McGrath P, Kay J, MacMurray SB, Dulberg C. The development of a tool to assess neonatal pain. Neonatal Netw 1993;12:59-66.

14) Stevens B, Johnston C, Petryshen P, Taddio A. Premature Infant Pain Profile: development and initial validation. Clin J Pain 1996;12:13-22.

15) Gibbins S, Stevens B. Mechanisms of sucrose and non-nutritive sucking in procedural pain management in infants. Pain Res Manag 2001;6:21-8.

16) Blass EM, Shah A. Pain-reducing properties of sucrose in hu man newborns. Chem Senses 1995;20:29-35.

17) Barr RG, Pantel MS, Young SN, Wright JH, Hendricks LA, Gravel $\mathrm{R}$. The response of crying newborns to sucrose: is it a "sweetness" effect? Physiol Behav 1999;66:409-17.

18) Kumari S, Datta V, Rehan H. Comparison of the efficacy of oral $25 \%$ glucose with oral $24 \%$ sucrose for pain relief during heel 
lance in preterm neonates: a double blind randomized controlled trial. J Trop Pediatr 2017;63:30-5.

19) Suhrabi Z, Taghinejad H, Valian K, Sayehmiri K, Taheri S. A comparative study on the efficacy of glucose and sucrose on the vaccination pain: a randomized controlled clinical trial. J Clin Diagn Res 2014;8:PC01-3.

20) Jatana SK, Dalal SS, Wilson CG. Analgesic effect of oral glucose in neonates. Med J Armed Forces India 2003;59:100-4.

21) Okan F, Coban A, Ince Z, Yapici Z, Can G. Analgesia in preterm newborns: the comparative effects of sucrose and glucose. Eur J Pediatr 2007;166:1017-24.

22) Skogsdal Y, Eriksson M, Schollin J. Analgesia in newborns given oral glucose. Acta Paediatr 1997;86:217-20.

23) Deshmukh LS, Udani RH. Analgesic effect of oral glucose in preterm infants during venipuncture: a double-blind, rando- mized, controlled trial. J Trop Pediatr 2002;48:138-41.

24) Ling JM, Quah BS, Van Rostenberghe $H$. The safety and efficacy of oral dextrose for relieving pain following venepuncture in neonates. Med J Malaysia 2005;60:140-5.

25) Carbajal R, Lenclen R, Gajdos V, Jugie M, Paupe A. Crossover trial of analgesic efficacy of glucose and pacifier in very preterm neonates during subcutaneous injections. Pediatrics 2002;110(2 Pt 1):389-93.

26) Blass EM, Shide DJ. Some comparisons among the calming and pain-relieving effects of sucrose, glucose, fructose and lactose in infant rats. Chem Senses 1994;19:239-49.

27) Isik U, Ozek E, Bilgen H, Cebeci D. Comparison of oral glucose and sucrose solutions on pain response in neonates. J Pain 2000;1:275-8. 